ARTICLE

Received 11 Oct 2013 | Accepted 21 Nov 2013 | Published 19 Dec 2013

\title{
Ultrashort single-walled carbon nanotubes in a lipid bilayer as a new nanopore sensor
}

Lei Liu', Chun Yang${ }^{1}$, Kai Zhao ${ }^{1}$, Jingyuan $\mathrm{Li}^{1} \&$ Hai-Chen Wu${ }^{1}$

An important issue in nanopore sensing is to construct stable and versatile sensors that can discriminate analytes with minute differences. Here we report a means of creating nanopores that comprise ultrashort single-walled carbon nanotubes inserted into a lipid bilayer. We investigate the ion transport and DNA translocation through single-walled carbon nanotube nanopores and find that our results are fundamentally different from previous studies using much longer single-walled carbon nanotubes. Furthermore, we utilize the new single-walled carbon nanotube nanopores to selectively detect modified 5-hydroxymethylcytosine in single-stranded DNA, which may have implications in screening specific genomic DNA sequences. This new nanopore platform can be integrated with many unique properties of carbon nanotubes and might be useful in molecular sensing such as DNA-damage detection, nanopore DNA sequencing and other nanopore-based applications.

\footnotetext{
${ }^{1}$ Key Laboratory for Biomedical Effects of Nanomaterials and Nanosafety, Institute of High Energy Physics, Chinese Academy of Sciences, 19B Yuquan Road, Beijing 100049, China. Correspondence and requests for materials should be addressed to H.-C.W. (email: haichenwu@ihep.ac.cn).
} 
A nanopore sensor can be regarded as a Coulter counter at the single-molecule scale ${ }^{1}$. The first major breakthrough in nanopore sensing occurred in the mid 1990s when $\alpha$-haemolysin $(\alpha \mathrm{HL})$ was used as a nanopore sensor. Kasianowicz et $a .^{2}{ }^{2}$ reported the investigation of the movement of polynucleotides through an $\alpha \mathrm{HL}$ nanopore, which marked the beginning of the era of nanopore DNA sequencing. At about the same time, Bayley and coworkers ${ }^{3-5}$ started to develop stochastic sensing of a wide variety of analytes based on $\alpha \mathrm{HL}$ and mutants. Another breakthrough came from the successful fabrication of solid-state (SS) nanopores with subnanometre control. Those pores are mostly drilled on silicon-based materials with ion-beam sculpting ${ }^{6}$ or electron-beam lithography ${ }^{7}$. The precise control of nanopore diameters and their inherent high durability have greatly expanded the scope of sensing substrates ${ }^{8}$. Recently, carbon nanotubes of micron length ${ }^{9}$, drilled graphene membranes ${ }^{10-12}$ and designed DNA nanostructures ${ }^{13}$ were also used as nanopores for DNA analysis. Despite the advances over the last two decades, to develop new sensors with unique functions such as high sensitivity and selectivity is still the central task in nanopore technology.

Here we report a means of creating nanopores that comprise ultrashort single-walled carbon nanotubes (SWCNTs) inserted into a lipid bilayer. We study the ion transport and singlestranded DNA (ssDNA) translocation through SWCNT nanopores and find that our results are fundamentally different from previous studies conducted with much longer SWCNTs ${ }^{9,14}$. The occurrence of exceptionally large conductance changes during ssDNA traversing SWCNT nanopores reveals that the ion mobilities inside SWCNTs are two to four times higher than the bulk mobility. Furthermore, we utilize the new SWCNT nanopores to selectively detect modified 5-hydroxymethylcytosine $(5 \mathrm{hmC})$ in ssDNA, which may have implications for high-throughput screening of genomic DNA fragments that contain $5 \mathrm{hmC} \mathrm{C}^{15}$. Our findings provide an effective and reliable strategy for constructing a new type of carbon-based sensors and demonstrate its potential for nucleic acid analysis. This new nanopore platform, integrated with many special properties of SWCNTs, is expected to be useful in molecular sensing and many other nanopore-based applications, such as ion channels ${ }^{16}$ and nanopore DNA sequencing ${ }^{17-19}$.

\section{Results}

Construction of SWCNT nanopores. The ultrashort SWCNTs with diameter of $0.8-2.0 \mathrm{~nm}$ and length of 5-10 $\mathrm{nm}$ were obtained by cutting long SWCNTs (Supplementary Fig. S1) with sonication in concentrated sulphuric acid/nitric acid (3/1) and subsequent size-exclusion separation on high-performance liquid chromatography (HPLC). The setup for protein nanopore research was adopted for the current study (Fig. 1a). The thickness of 1,2-diphytanoyl-sn-glycero-3-phosphocholine ( $\mathrm{DPhPc}$ ) bilayer is $\sim 5 \mathrm{~nm}$; therefore, the ideal length of the SWCNTs for our nanopore system is approximately between 5 and $10 \mathrm{~nm}$, which was proved by atomic force microscopy characterization and the insertion experiments with different HPLC fractions (Supplementary Figs S2-S5). However, there is still a remaining challenge for the nanopore system to be viable, that is, how to effectively insert a single SWCNT into the lipid bilayer. After several unsuccessful attempts, we eventually designed a microinjection probe that could reliably insert sub-10 nm SWCNTs into lipid bilayer (Fig. 1b, Supplementary Fig. S6 and Supplementary Movies 1 and 2). On insertion, the tip of the probe with an injection rate of $1.0 \mu \mathrm{l} \mathrm{min}{ }^{-1}$ was brought in close proximity to lipid bilayer but without touching it. After a current jump caused by the insertion occurred, the probe was slowly taken away from the bilayer to avoid any further interference. About two-thirds of the insertions afforded stable current signals straightway and the rest underwent a stabilizing period varying from several seconds to a few minutes (Supplementary Fig. S7). Less than $5 \%$ of the inserted SWCNTs showed persistent unstable current levels and were thus abandoned.

Ion transport inside different SWCNT nanopores. We first measured the conductance of SWCNT-based nanopores. Among the 180 nanopores that have been recorded, all the current traces show stable baseline and high signal-to-noise ratio (Supplementary Fig. S8). Three typical current-voltage $(I-V)$ curves and their corresponding conductance values are shown in Fig. 2a-c. We did not observe any stochastic pore blocking by ions as reported by Strano and coworkers ${ }^{14}$, probably because the SWCNTs used in our experiments are $\sim 100,000$ times shorter than theirs and the voltage applied in our SWCNT nanopores is also much lower than the threshold voltage in their measurements. The conductance of all the SWCNT nanopores we measured spans four orders of magnitude and can be roughly categorized into three groups (Fig. 2d). Group 1 has enormously large conductance of 60-100 nS, and group 3 has relatively small conductance of $0.1-2 \mathrm{nS}$, whereas group 2 of intermediate values $(3-24 \mathrm{nS})$ is sandwiched in the middle (standard buffer conditions: $1 \mathrm{M} \mathrm{KCl}, 10 \mathrm{mM}$ Tris, $\mathrm{pH}$ 8.0). The large conductance group contains all metallic SWCNTs, as suggested in a similar scenario in ref. 9. Conductance measurements using ultrashort pure metallic SWCNTs (IsoNanotubes-M) also afforded conductance values in the similar ranges to group $\mathbf{1}$.

The SWCNTs in group $\mathbf{2}$ and $\mathbf{3}$ are semiconductors, and the ones in group 2 have larger diameters that enable them to pass ssDNA, which we observed experimentally (Fig. 2d, red histogram). For electrolyte inside a nanotube with diameter of 1-2 $\mathrm{nm}$ and length of 5-10 nm, the calculated ionic conductance is between 1.18 and $9.40 \mathrm{nS}$ without the consideration of freeenergy barrier (Supplementary Methods). However, many measured conductance values in group 2 exceed this predicted range (Fig. 2d). This has prompted us to investigate whether there is a different ion transport mechanism in this new nanopore system, as will be discussed vide infra. Similarly, according to the a

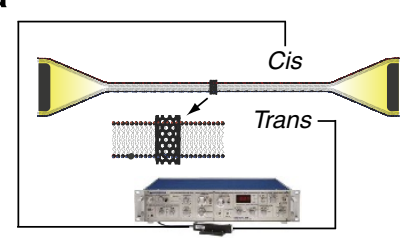

b

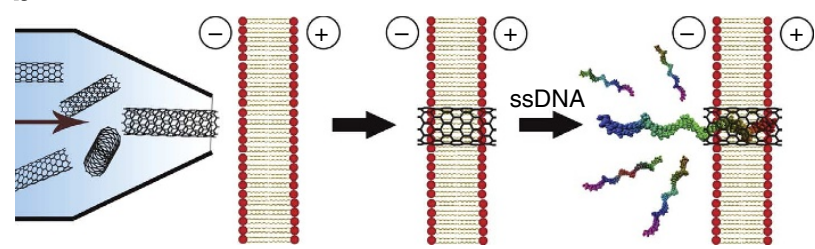

Figure 1 | Schematic illustration of the setup and workflow. (a) A patch-clamp amplifier was adopted to measure the currents through SWCNT nanopores. (b) Illustration of the insertion of a SWCNT into lipid bilayer with a micro-injection probe and ssDNA translocation through the inserted SWCNT. 
a

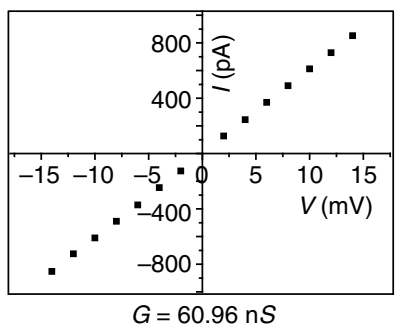

b

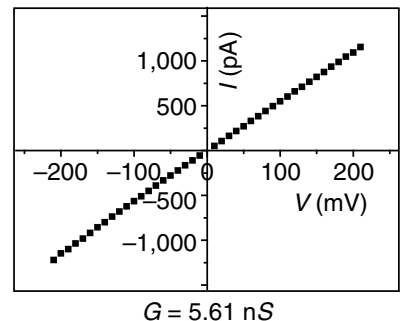

c

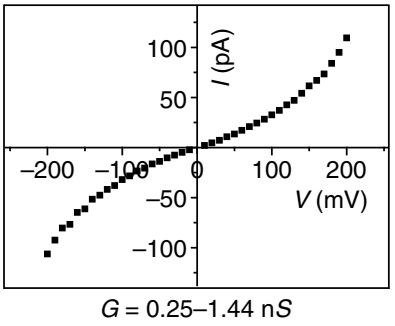

d

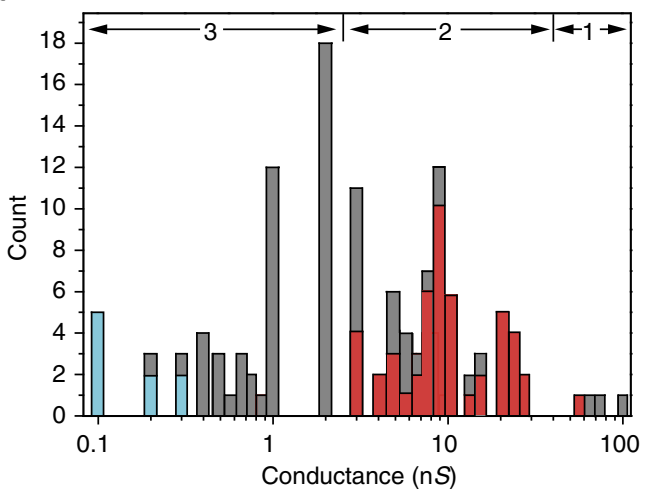

e

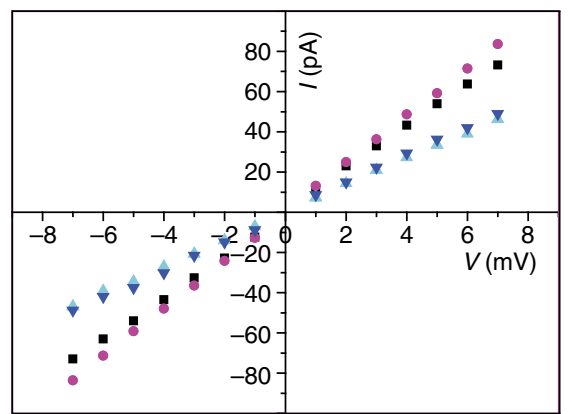

Figure 2 | Categorization of SWCNT nanopores. (a-c) Representation of $I-V$ curves and conductance value of a SWCNT nanopores of group 1-3, respectively. Data were acquired in the buffer of $1 \mathrm{M} \mathrm{KCl}$ and $10 \mathrm{mM}$ Tris, $\mathrm{pH}$ 8.0. (d) Conductance distribution of SWCNT nanopores categorized in three groups 1-3. The SWCNTs that passed ssDNA molecules are marked in red (the unmarked tubes in group 2 were not tested for DNA translocation). The SWCNTs that showed nonlinear I-V curves are marked in turquoise. (e) I-V curves of a SWCNT nanopore in group $\mathbf{2}$ under four different pH conditions (pH 10.0, circle; $\mathrm{pH}$ 8.0, square; $\mathrm{pH} 3.44$, inverted triangle; $\mathrm{pH}$ 2.89, triangle). The experiments were repeated in six different SWCNT nanopores.

conductance calculation, it appears that SWCNTs in group 3 have diameters $<1 \mathrm{~nm}$. This has been confirmed by Raman spectroscopy characterization (Supplementary Fig. S9 and Supplementary Table S1) and ssDNA translocation experiments, as none of the SWCNTs in group 3 could pass ssDNA. Another notable result obtained in group 3 is when the conductance of SWCNT nanopores decreases to $0.1-0.3 \mathrm{n} S$, the $I-V$ curves become nonlinear but remain symmetrical for positive and negative potentials (Fig. 2c,d, turquoise histograms). A plausible explanation could be found in a simulation study by Peter and Hummer ${ }^{20}$, which shows that when ions transport through a nanopore with diameter $<1 \mathrm{~nm}$, the hydration layers around the ions need to be stripped off due to the spatial constrains, creating a large energetic penalty. It was previously shown that the ionic conductance through $2-\mu \mathrm{m}$-long SWCNTs was dependent on the $\mathrm{pH}$ of buffer solutions ${ }^{9}$. We also measured the ionic conductance of ultrashort SWCNT nanopores under different $\mathrm{pH}$ values (Fig. 2e). The results confirmed that the charged end groups (carboxylic acid residues) played a vital role in ion conduction through nanotubes.

ssDNA translocation through SWCNT nanopores. Next we examined ssDNA translocation through SWCNT nanopores, taking advantage of the ultrahigh sensitivity of PCR for detecting small numbers of molecules. We used 120-nucleotide oligomers (DNA1 in Supplementary Table S2. PCR protocol shown in Supplementary Table S3) with sequences predicted to have minimal secondary structures ${ }^{2}$. DNA solution was added in the cis compartment (final concentration $1.0 \mu \mathrm{M}$ ), which was connected to ground, and DNA molecules were driven through SWCNT nanopores under positive potentials. Three different scenarios could be observed when DNA oligomers were added for translocation through various SWCNT nanopores. First, the current trace remained quiescent if the conductance of the SWCNT nanopore was relatively small $(\leq 2.0 \mathrm{n} S)$. Second, transient current blockades of $\sim 20 \mathrm{pA}$ started to occur frequently (Supplementary Fig. S10). Third, remarkably large current blockades appeared immediately after the addition of DNA (Fig. 3a). We identified these large-amplitude blockades as DNA translocation events by quantitative PCR analysis of the translocated DNA molecules (Supplementary Fig. S11). The short-lived 20-pA blockades are ascribed to DNA colliding with the ends of SWCNTs, as PCR showed negative results.

It is important to note that our observation of DNA translocation through ultrashort SWCNTs $(5-10 \mathrm{~nm})$ in $1 \mathrm{M}$ $\mathrm{KCl}$ is entirely different from an earlier report ${ }^{9}$, in which DNA translocation through $2-\mu \mathrm{m}$-long SWCNTs was shown to be current-increasing spikes rather than current blockades. The mechanism accounting for this drastic difference requires further investigation. For comparison with other nanopore systems, we performed detailed studies of ssDNA translocation through different SWCNT nanopores (Fig. 3b, and Supplementary Figs S12 and S13). Two distinctive features are observed and discussed below.

First, the voltage required to drive DNA through SWCNT nanopores $(10-40 \mathrm{mV})$ is much lower than that for $\alpha \mathrm{HL}$ $(\geq 80 \mathrm{mV})$ (refs 21,22$)$ and SS-nanopores $(\geq 100 \mathrm{mV}$ when $d_{\text {pore }} \approx 10 \mathrm{~nm}$ ) (ref. 23). The diameter of SWCNTs in our experiments is close to or even smaller than that of the other two types of nanopores, which means the entropic barrier for DNA translocation in SWCNT nanopore should be similar to or higher than that of other nanopores. The difference in driving voltage must arise from DNA-nanopore interactions, which 
a

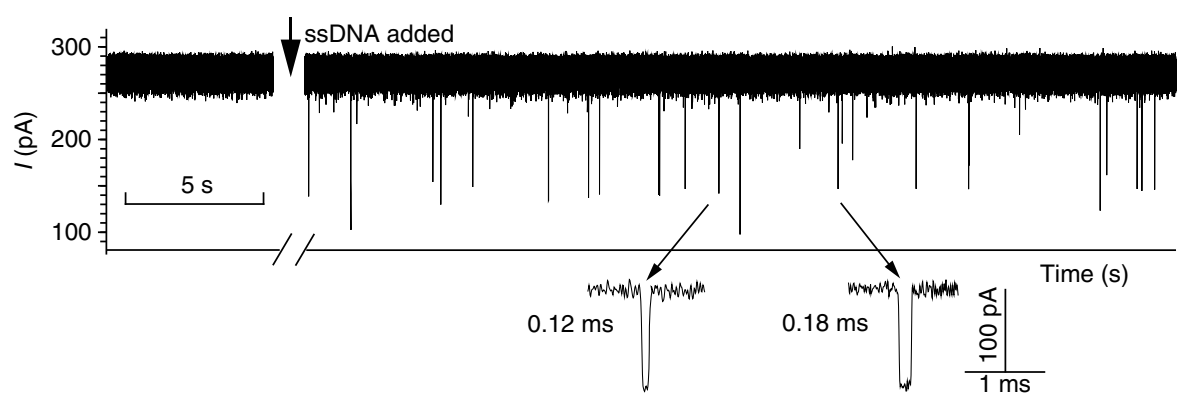

b

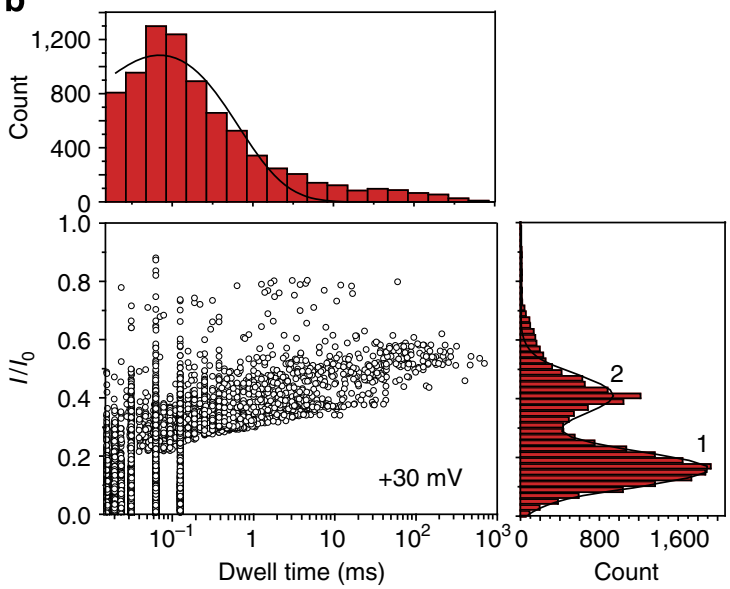

C

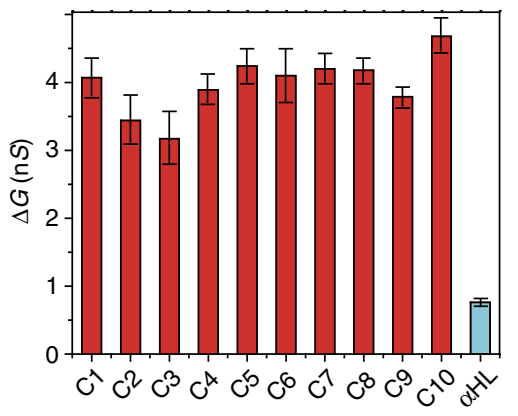

Figure 3 | Translocation of ssDNA through SWCNT nanopores. (a) Typical ionic current signal recorded when DNA1 (final concentration $1.0 \mu M$ ) was added to the cis side of a SWCNT nanopore $\left(G_{C N T}=8.97 \mathrm{nS}\right)$. The capture rate is $\sim 2,300$ events per $\mathrm{h}$. Data were acquired in the buffer of $1 \mathrm{M}$ $\mathrm{KCl}$ and $10 \mathrm{mM}$ Tris, $\mathrm{pH} 8.0$, with the transmembrane potential held at $+30 \mathrm{mV}$. (b) Scatter plot of the events (normalized current blockage $/ / I_{0}$ versus event durations) in a. The dwell-time histogram was constructed using the data points with $I / I_{0}>0.3$. The solid line in dwell-time histogram is a mono-exponential fit to the data. Peak 1 in $I / I_{0}$ histogram represents SWCNT-DNA colliding events and peak 2 represents DNA translocation events. The lines on peaks 1 and 2 are Gaussian fit to the histogram. (c) Comparison of conductance change values $(\Delta G)$ of DNA translocation through SWCNT nanopores (C1-10) and $\alpha \mathrm{HL}$. The error bars are defined as s.d. of at least three experiments. The conductance values of the nanopores are as follows: $G_{C N T}$ (1-10) $8.30,8.39,8.54,8.65,9.63,16.41,16.75,24.59,25.28$ and $60.75 \mathrm{nS} ; \mathrm{G}_{\alpha \mathrm{HL}}=0.93 \mathrm{nS}$

involve multiple weak force interactions within $\alpha \mathrm{HL}$ and SSnanopores, whereas in SWCNT nanopores DNA molecules are significantly attracted into SWCNTs through favourable interactions of the bases with the SWCNT pore wall ${ }^{24,25}$.

Second, the conductance change $(\Delta G)$ caused by DNA traversing SWCNT nanopores are exceptionally large (Fig. 3a) compared with that in $\alpha \mathrm{HL}$. The $\Delta G$ values of DNA translocation of SWCNT nanopores (ten examples) $\alpha \mathrm{HL}$ under identical buffer conditions are listed in Fig. 3c. When a DNA molecule enters the nanopore, it causes two competing effects. On one hand, the conductance is decreased because of the steric exclusion of both cations and anions by $\mathrm{DNA}^{26}$; on the other hand, the counterions shielding the phosphate groups on the DNA backbone are introduced into the nanopore and thus enhance the conductance. According to a previously established model ${ }^{27}$, the conductance change due to the DNA translocation through SWCNT nanopores, $\Delta G_{\mathrm{CNT}}$, can be expressed as:

$$
\Delta G_{C N T}=\frac{1}{L_{\mathrm{CNT}}}\left(-\frac{\pi}{4} d_{\mathrm{ssDNA}}^{2}\left(\mu_{\mathrm{K}}+\mu_{\mathrm{Cl}}\right) c_{\mathrm{KCl}} e+\mu_{\mathrm{K}}^{*} q_{\mathrm{DNA}}^{*}\right)
$$

where $L_{\mathrm{CNT}}$ represents the length of the SWCNT, $d_{\text {ssDNA }}$ $(\sim 1.1 \mathrm{~nm})$ is the diameter of ssDNA, $\mu_{\mathrm{K}}^{*}$ is the effective electrophoretic mobility of potassium ions moving along the DNA and $q_{\mathrm{DNA}}{ }^{*}$ is the effective charge on the DNA per unit length, which is assumed to be constant. For the ten SWCNT nanopores, we obtained the mean $\Delta G_{\mathrm{CNT}}=3.98 \pm 0.43 \mathrm{nS}$ and $L_{\mathrm{CNT}}=5-10 \mathrm{~nm}$, whereas for $\alpha \mathrm{HL}$ nanopore $\Delta G_{\alpha \mathrm{HL}}=0.75 \mathrm{nS}$ and $L_{\alpha \mathrm{HL}} \approx 10 \mathrm{~nm}$. In high-ionic-strength electrolyte solution inside nanopores $\left(c_{\mathrm{KCl}}=1 \mathrm{M}, D_{\text {pore }}>1.1 \mathrm{~nm}\right), \mu_{\mathrm{K}} \approx \mu_{\mathrm{Cl}}$. If we take an approximation that $\mu_{\mathrm{K}}^{*}$ equals the ion mobility inside nanopores $\mu_{\mathrm{K}}$ (ref. 27), we can obtain $\Delta G_{\text {pore }} \propto \mu_{\mathrm{K}} / L_{\text {pore }}$, that is, $\mu_{\mathrm{K}} \propto \Delta G_{\text {pore }} L_{\text {pore. }}$ By comparing these two groups of data, we can conclude that the ion mobilities in SWCNT nanopores are enhanced roughly two- to four times higher than that in $\alpha \mathrm{HL}$, where ion mobilities are shown to be the same as bulk mobility. This conclusion is in qualitative agreement with a molecular dynamic simulation study by Peter and Hummer ${ }^{20}$, which showed an $\sim 50 \%$ increase in ion mobilities inside SWCNTs. Moreover, our deduced value seems to nicely coincide with the measurements of ion mobilities through membranes that contain a large number of SWCNT pores in a recent report ${ }^{16}$. However, caution must be taken in the comparison because of the distinct differences between the two systems, including SWCNT diameter (1.1-1.8 nm versus $0.9 \mathrm{~nm})$ and length $(5-10 \mathrm{~nm}$ versus $5 \mu \mathrm{m})$, salt concentration ( $1 \mathrm{M}$ versus $6 \mathrm{mM})$ and single-channel versus ensemble measurements and so on.

Identification of chemically modified $5 \mathrm{hmC}$ in ssDNA. To explore the potential advantages of SWCNT nanopores, we carried out DNA-sensing experiments for identifying $5 \mathrm{hmC}$ in the sequence. $5 \mathrm{hmC}$, now considered the sixth base, has been increasingly recognized not only as an intermediate in DNA demethylation but also for having important roles in epigenetic 
reprogramming and regulation of tissue-specific gene expres$\operatorname{sion}^{15}$. However, conventional bisulphite sequencing cannot discriminate $5 \mathrm{hmC}$ from 5-methylcytosine (5mC). Thus, various methods have been and are being developed to differentiate these two bases in DNA sequences ${ }^{28-31}$. Here we employed a strategy of a combination of chemical modification and nanopore sensing to selectively detect $5 \mathrm{hmC}$ in a short ssDNA. The two-step modification is bio-orthogonal and highly specific to $5 \mathrm{hmC}$ (Fig. 4a and Supplementary Fig. S14). We then used SWCNT nanopores to screen the modified DNAs (Supplementary Table S2), hoping to exploit their intrinsic high sensitivity. A characteristic type of current signature was observed when benzoimidazole (Bzim)-modified 5hmC-containing DNA2 was translocated through SWCNT nanopores (Fig. 4b). In this multilevel blockade, level 1 state represented either $3^{\prime}$ - or $5^{\prime}$-end of DNA2-Bzim entering SWCNT nanopore, whereas the Bzimmodified part had not yet reached the nanopore. Level 2 with a shape of current-increasing spike immediately followed level 1 and represented the Bzim part entering and sliding through the nanopore. Finally, the current dropped to level 3, which was caused by the translocation of the rest of DNA2-Bzim, before returning to the full open-pore level (Fig. 4b). We plotted the histograms of the conductance difference between the three levels $(\Delta G 1-3)$ and the open-pore level (Fig. 4c). It was seen that $\Delta G 2$ varied in a wider range than $\Delta G 1$ and $\Delta G 3$, while the latter two always remained at about the same height (for examples, see Supplementary Fig. S15). The frequency of signature events $\left(f_{\text {sig }}\right)$ was found to be proportional to the DNA2-Bzim concentration range from 10 to $100 \mathrm{nM}$ (Supplementary Fig. S16a). The detection limit might be lowered for several orders of magnitude when asymmetric electrolyte conditions are used ${ }^{32-34}$. It should be noted that the proportion of the characteristic events is $\sim 10 \%$ of total events, probably because DNA degradation during the $5 \mathrm{hmC}-\mathrm{DNA}$ oxidation $\mathrm{step}^{28}$ generated unmodified

a

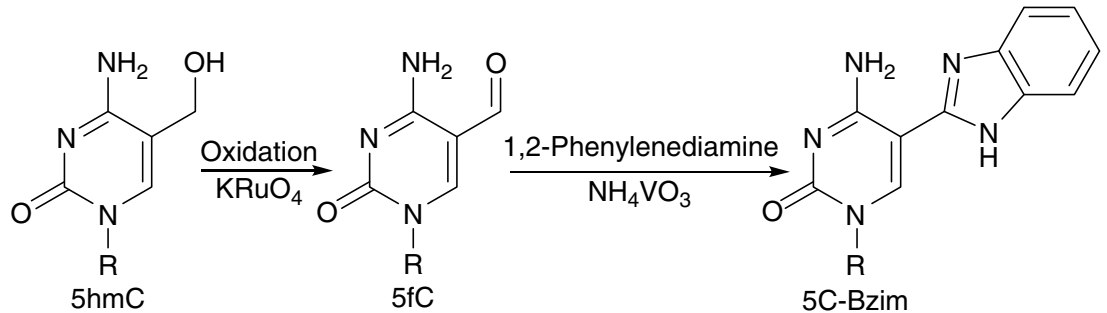

b

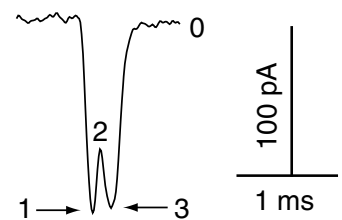

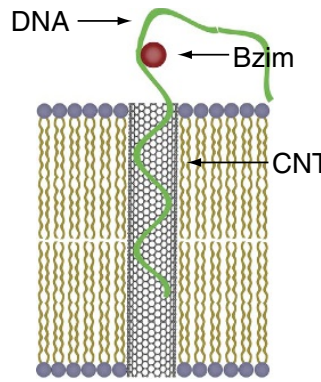

Level 1

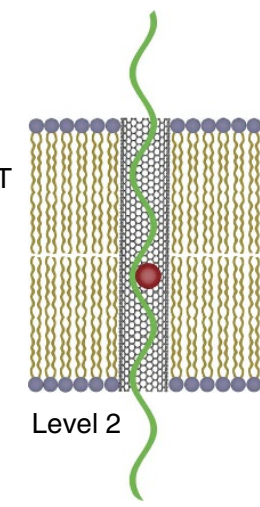

d

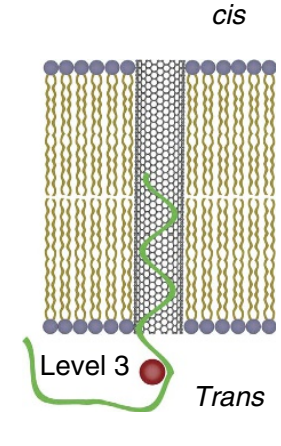

Trans
C

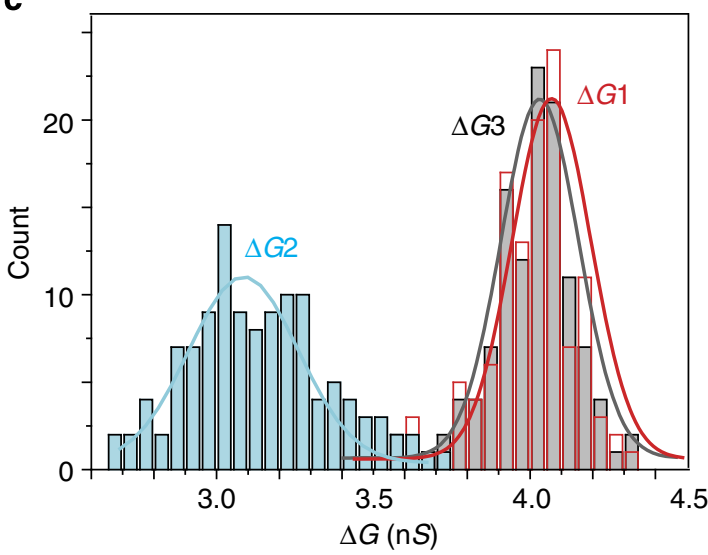

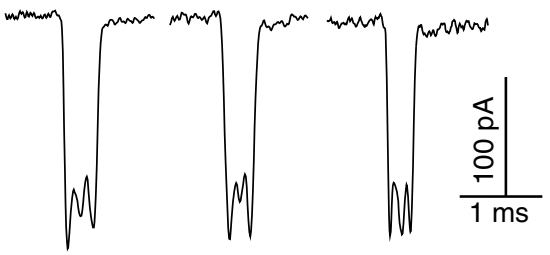

Figure 4 | Detection of $5 \mathrm{hmC}$ in ssDNA using SWCNT nanopore. (a) $5 \mathrm{hmC}$ modification scheme: oxidation ${ }^{28}$ of $5 \mathrm{hmC}$ to $5 \mathrm{fC}$ followed by oxidative condensation with 1,2-phenylenediamine to afford 5C-Bzim. The R group is DNA. (b) A typical multilevel current signature and diagram showing molecular mechanism of DNA2-Bzim translocation through SWCNT nanopore. Level 1: DNA2-Bzim entering the nanopore before Bzim-modified part reaches the pore; level 2: the Bzim part entering and sliding through the nanopore; level 3: translocation of the rest of DNA2-Bzim. The mean duration of 118 singlespike events is $406 \pm 111 \mu \mathrm{s}$. (c) Histograms of the conductance differences between level 1-3 and the open-pore level $G_{0}$ in $\mathbf{b}$ (118 events in total). (d) New type of current signature caused by the translocation of DNA5-Bzim, which contains double $5 \mathrm{hmC}$ sites. The mean duration of 110 double-spike events is $536 \pm 161 \mu \mathrm{s}$. All the data were acquired in the buffer of $1 \mathrm{M} \mathrm{KCl}$ and $10 \mathrm{mM}$ Tris, $\mathrm{pH} 8.0$, with the transmembrane potential held at $+40 \mathrm{mV}$. 
DNA fragments that retained in the final solution and contributed to the total number of DNA translocation events.

To confirm that the presence of current signature was due to the modified $5 \mathrm{hmC}$, we carried out control experiments in which $5 \mathrm{hmC}$ in DNA2 was replaced with $5 \mathrm{mC}$ or cytosine $(\mathrm{C})$. The results showed that $5 \mathrm{mC} / \mathrm{C}$-containing DNAs were inert under the modification conditions and no characteristic current signature was observed in those control groups. In addition, we also examined DNA5, which contains two 5hmC sites (Supplementary Table S2). Translocation of DNA5-Bzim afforded a new type of current signature with double currentincreasing spikes and its frequency was proportional to the DNA5-Bzim concentration between 10 and $100 \mathrm{nM}$ (Fig. 4d and Supplementary Fig. S16b). This result further substantiated the correlation of the spikes in current signature with the modified $5 \mathrm{hmCs}$ in DNA.

\section{Discussion}

It has been demonstrated in literature that modified ssDNA could produce characteristic current signals when passing through $\alpha \mathrm{HL}^{35}$. However, those modifiers were generally very bulky groups, which caused a further current blockade on top of the DNA-occupying level during translocation. To reveal whether Bzim-modified DNA can generate any kind of 'signature events' when translocated through $\alpha \mathrm{HL}$, we carried out DNA2-Bzim traversing $\alpha \mathrm{HL}$ experiments under otherwise identical conditions for SWCNT nanopores. The results are shown in Supplementary Fig. S15b. It is interesting to see that DNA2-Bzim did produce a small fraction of 'unusual current events' ( $<10 \%$ of total events) during translocation through $\alpha \mathrm{HL}$. Those events all have a transient medium-level blockade before the DNA translocation level. Previous studies by Butler et al. ${ }^{36}$ had shown that this medium-conductance state was caused by DNA occupancy in the cis vestibule of $\alpha \mathrm{HL}$. It seems that the Bzim modification is not bulky enough to cause any conductance change during DNA translocation, but it somehow delays the process of DNA lingering in the cis vestibule and thus produces a marginally discernible conductance state in the events. However, the minor difference between these current events is not determinative enough for the identification of $5 \mathrm{hmC}$ in ssDNA using $\alpha \mathrm{HL}$. Interestingly, a very recent report also described the detection of chemically modified $5 \mathrm{hmC}$ in immobilized ssDNA with $\alpha \mathrm{HL}$ nanopore 37 . Although modification of $5 \mathrm{hmC}$ with different chemical groups could be discriminated by residual current levels in nanopore analysis, this strategy suffered from the fact that the $5 \mathrm{hmC}$ has to be at a specific position in the ssDNA to be detected.

In comparison with $\alpha \mathrm{HL}$, identification of modified $5 \mathrm{hmC}$ in ssDNA through SWCNT nanopores can be achieved with high confidence owing to the characteristic current signals. Although the locations of $5 \mathrm{hmC}$ cannot be quantitatively determined from the signature events due to the exceedingly fast DNA translocation through SWCNT nanopores, this new sensor offers a simple and powerful tool for: (i) high-throughput screening of genomic DNA fragments that contain $5 \mathrm{hmC}$ and (ii) differentiating $5 \mathrm{mC}$ and $5 \mathrm{hmC}$ in bisulphite sequencing. This strategy can detect any $5 \mathrm{hmC}$ site in the DNA strand regardless of the local sequence context and might be extended to many other useful applications in chemistry and biology.

The successful fabrication of ultrashort SWCNT-based nanopores is another exciting advance in the development of nanopore technology. Currently, the major obstacle for SWCNT nanopore development is the complex composition of the as-grown CNT and precise manipulation of individual SWCNTs. The recent successes in the controlled growth of SWCNTs with single chirality $^{38}$ or structure-specific separation of CNT mixtures ${ }^{39}$ might significantly improve the efficiency and reliability of the fabrication of SWCNT nanopores. Although the current device is based on lipid bilayer membrane, which is intrinsically fragile yet convenient to manipulate, the technology of embedding SWCNTs in solid support is readily available ${ }^{40}$. Many of the peculiar properties of CNTs can be integrated into nanopore to achieve special goals unobtainable from other nanopore systems. For instance, SWCNTs of different diameters with subangstrom precision provide a large repertoire of candidates for constructing various artificial ion channels, which nicely complements a recently reported approach that forms transmembrane channels in lipid bilayers with DNA-based nanostructures ${ }^{13}$. For another example, SWCNT nanopores of different chiralities may have special recognitions for different small molecules such as 20 amino acids, which provides the basis of protein sequencing. More generally, the electronic structure ${ }^{41}$ and ferromagnetic anisotropy of SWCNTs ${ }^{42}$ would allow the incorporation of gate voltage and magnetic interactions into the platform of SWCNT nanopores and investigate the controlled mass transport at the single-molecule level. The simplicity of the environment inside SWCNT nanopores and the perfect uniformity of the graphitic wall of SWCNTs would provide a great platform for developing many other nanopore-related applications, especially for nanopore DNA sequencing and DNA-damage sensing.

\section{Methods}

Materials. DPhPc was purchased from Avanti Polar Lipids (Beijing distributor). Wild type D8H6 $\alpha \mathrm{HL}$ was produced by expression in BL21 (DE3) pLysS Escherichia coli cells and self-assembled into heptamers ${ }^{34}$. DNA1, DNA6-8 and $(\mathrm{GT})_{29}$ oligomer were purchased from Sangon Biotech, Shanghai, and DNA2-5 oligomers were purchased from Takara Bio Inc. (Dalian). SWCNTs (90\% purity) were purchased from Timesnano (Chengdu) and further purified in our laboratory The SWCNTs were synthesized by chemical vapour deposition method, with the length of $5-30 \mu \mathrm{m}$ and diameter of 1-2 nm. IsoNanotubes-M (metallic SWCNTs) was purchased from Nanointegris.

Preparation of short SWCNTs. The short SWCNTs were prepared in a two-step process. One hundred milligrams of the purified SWCNTs were suspended in $24 \mathrm{~m}$ mixture of concentrated $\mathrm{H}_{2} \mathrm{SO}_{4} / \mathrm{HNO}_{3}(3 / 1)$ in a 250 -ml conical flask and sonicated in a water bath for $48 \mathrm{~h}$ at $35-40{ }^{\circ} \mathrm{C}$ (ref. 43 ). The suspension was then diluted with $200 \mathrm{ml}$ of water and neutralized with $6 \mathrm{M} \mathrm{NaOH}$ solution. The relatively long SWCNTs ( $>300 \mathrm{~nm}$ by scanning electron microscopy, CNT1) were collected on a 100-nm pore size filter membrane (PTFE; Millipore) and the shorter SWCNTs (CNT2) were collected in the filtrate. The solution was taken for centrifugal ultrafiltration ( $3 \mathrm{kDa}$ UFC900308; Millipore) for $40 \mathrm{~min}$ and then Milli$\mathrm{Q}$ water was added to dilute the solution for further centrifugal ultrafiltration. The washing and ultrafiltration were repeated three times before water was completely removed to afford a black powder as the product.

Length separation of short SWCNTs. We followed the protocol developed by Zheng and coworkers ${ }^{44}$ to conduct the length separation of short SWCNTs on HPLC. Both SWCNT samples of CNT1 and CNT2 were dispersed with DNA oligomer $(\mathrm{GT})_{29}$ before HPLC separation. In a typical procedure, $0.8 \mathrm{mg}$ of SWCNTs was suspended with $0.8 \mathrm{mg}(\mathrm{GT})_{29}$ in $1 \mathrm{ml}$ aqueous buffer solution (40 mM Tris, pH 7.0, 0.5 mM EDTA and $0.2 \mathrm{M} \mathrm{NaCl}$ ). The mixture was kept in an ice-water bath and sonicated (Sonics, vibra cell) for $90 \mathrm{~min}$. After sonication, the mixture was then centrifuged (Sigma $3 \mathrm{~K} 15$ ) for $90 \mathrm{~min}$ at $16,000 \mathrm{~g}$ to remove insoluble material, resulting in a DNA-dispersed SWCNTs solution. Length separation by HPLC was carried out using size-exclusion columns (COSMOSIL CNT, Nacalai Tesque Inc). We used two types of columns (dimension: $7.5 \mathrm{~mm} \times 300 \mathrm{~mm}$, pore size: 300 and $1,000 \AA$ ) connected in series to separate both CNT1 and CNT2 samples. Dispersed DNA-SWCNT solutions were filtrated through a $0.22-\mu \mathrm{m}$ membrane and loaded $(\sim 0.5 \mathrm{ml})$ onto the column series. The hybrid was eluted with pH 7.0 buffer containing $40 \mathrm{mM}$ Tris, $0.5 \mathrm{mM}$ EDTA and $0.2 \mathrm{M} \mathrm{NaCl}$. Each fraction was collected at every 1-min interval.

Micro-injection probe. We attempted several approaches to solve the SWCNT insertion problem, including continuous stirring (up to $2 \mathrm{~h}$ ), agarose gel probe ${ }^{45}$ and glass probe ${ }^{46}$, but none of them worked. Eventually, we designed a microinjection probe that could reliably insert ultrashort SWCNTs into lipid bilayer. The micro-injection probe is controlled with a micromanipulator (Narishige Scientific Instrument Lab., Japan). The probe is composed of two main parts: a homemade 
micro-injector and a glass needle. The needle is made from a taper glass capillary tube stretched by a capillary puller. The opening of the needle has outer diameter of $\sim 20 \mu \mathrm{m}$ and an inner diameter of $3-5 \mu \mathrm{m}$.

\section{General procedure for 5hmC-DNA modification}

Oxidation. About $20 \mu \mathrm{g}$ of synthetic 5hmC-DNA (DNA2 or DNA5) was made up to $24 \mu \mathrm{l}$ with $\mathrm{NaOH}\left(0.05 \mathrm{M}\right.$ final concentration) on ice, $1 \mu \mathrm{l}$ of a $\mathrm{KRuO}_{4}$ (Alfa Aesar) solution ( $15 \mathrm{mM}$ in $0.05 \mathrm{M} \mathrm{NaOH}$ final concentration) was added and the reaction was held on ice for $1 \mathrm{~h}$, with occasional vortexing ${ }^{28}$. Next, $100 \mu \mathrm{l}$ buffer (100 mM HEPES, $1 \mathrm{mM}$ EDTA, $\mathrm{pH}$ 7.7) was added to the mixture. The reaction mixture was purified with a Micro Bio-SpinP6 column (Bio-Rad)

Condensation. Fifteen microlitres ammonium metavanadate $(5.31 \mathrm{mM}$ ) (Alfa Aesar, Tianjin) and $o$-phenylenediamine $(91.15 \mathrm{mM})$ containing buffer solution (100 mM HEPES, $1 \mathrm{mM}$ EDTA, pH 7.7) was added to 5-formylcytosine DNA solution from the oxidation step. The reaction was held at room temperature for $2 \mathrm{~h}$, with vortexing. Finally, the mixture was purified with a Micro Bio-Spin P6 column. The recovery rate of the modified $5 \mathrm{hmC}-\mathrm{DNA}$ is $\sim 50 \%$ based on the comparison of the DNA concentrations before and after modification (the reaction yields are assumed to be $100 \%$ ).

Single-channel current recording. A bilayer of DPhPc was formed across an aperture $100-150 \mu \mathrm{m}$ in diameter in a $20-\mu \mathrm{m}$-thick polycarbonate film (Goodfellow, Malvern, PA) that divided a planar bilayer chamber into two compartments, cis and trans. Both compartments contained $1 \mathrm{ml}$ of buffer. SWCNTs and DNA were added to the cis compartment, which was connected to ground. The trans compartment was connected to the head-stage of the amplifier. All experiments were carried out in $1 \mathrm{M} \mathrm{KCl}, 10 \mathrm{mM}$ Tris, $\mathrm{pH} 8.0$, at $22.5 \pm 2{ }^{\circ} \mathrm{C}$, unless otherwise stated. Currents were recorded by connecting a patch-clamp amplifier (Axopatch 200B; Axon instruments, Foster City, CA) to an external eight-pole Bessel low-pass filter (type 901, Frequency Devices) operating at $29.9 \mathrm{kHz}$, and sampled at $100 \mathrm{kHz}$ by a computer equipped with a Digidata $1440 \mathrm{~A} / \mathrm{D}$ converter (Axon instruments). For $5 \mathrm{hmC}-\mathrm{DNA}$-sensing experiments, data were acquired at $100 \mathrm{kHz}$ with a 10-kHz low-pass Bessel filter.

Data analysis. Current traces were analysed with Clampfit 10.2 software (Axon Instruments). Events were detected using the Event Detection feature and used to construct amplitude and dwell-time histograms. Origin (Microcal, Northampton, MA) and Clampfit were used for histogram construction, curve fitting and graph presentation. Adobe Illustrator was used for making figures.

\section{References}

1. Coulter, W. H. Means for counting particles suspended in a fluid. U.S. Patent 2,656,508 (1953).

2. Kasianowicz, J. J., Brandin, E., Branton, D. \& Deamer, D. W. Characterization of individual polynucleotide molecules using a membrane channel. Proc. Natl Acad. Sci. USA 93, 13770-13773 (1996).

3. Gu, L. Q., Braha, O., Conlan, S., Cheley, S. \& Bayley, H. Stochastic sensing of organic analytes by a pore-forming protein containing a molecular adapter. Nature 398, 686-690 (1999).

4. Braha, O. et al. Simultaneous stochastic sensing of divalent metal ions. Nat. Biotechnol. 18, 1005-1007 (2000).

5. Movileanu, L., Howorka, S., Braha, O. \& Bayley, H. Detecting protein analytes that modulate transmembrane movement of a polymer chain within a single protein pore. Nat. Biotechnol. 18, 1091-1095 (2000).

6. Li, J. et al. Ion-beam sculpting at nanometre length scales. Nature 412, 166-169 (2001)

7. Storm, A. J., Chen, J. H., Ling, X. S., Zandbergen, H. W. \& Dekker, C. Fabrication of solid-state nanopores with single-nanometre precision. Nat. Mater. 2, 537-540 (2003).

8. Dekker, C. Solid-state nanopores. Nat. Nanotech. 2, 209-215 (2007).

9. Liu, H. et al. Translocation of single-stranded DNA through single-walled carbon nanotubes. Science 327, 64-67 (2010).

10. Garaj, S. et al. Graphene as a subnanometre trans-electrode membrane. Nature 467, 190-193 (2010).

11. Merchant, C. A. et al. DNA translocation through graphene nanopores. Nano Lett. 10, 2915-2921 (2010).

12. Schneider, G. F. et al. DNA translocation through graphene nanopores. Nano Lett. 10, 3163-3167 (2010).

13. Langecker, M. et al. Synthetic lipid membrane channels formed by designed DNA nanostructures. Science 338, 932-936 (2012).

14. Lee, C. Y., Choi, W., Han, J.-H. \& Strano, M. S. Coherence resonance in a single-walled carbon nanotube ion channel. Science 329, 1320-1324 (2010).

15. Branco, M. R., Ficz, G. \& Reik, W. Uncovering the role of 5hydroxymethylcytosine in the epigenome. Nat. Rev. Genet. 13, 7-13 (2012).
16. Wu, J., Gerstandt, K., Zhang, H., Liu, J. \& Hinds, B. J. Electrophoretically induced aqueous flow through single-walled carbon nanotube membranes. Nat. Nanotech. 7, 133-139 (2012).

17. Clarke, J. et al. Continuous base identification for single-molecule nanopore DNA sequencing. Nat. Nanotech. 4, 265-270 (2009).

18. Cherf, G. M. et al. Automated forward and reverse ratcheting of DNA in a nanopore at 5-angstrom precision. Nat. Biotechnol. 30, 344-348 (2012).

19. Manrao, E. A. et al. Reading DNA at single-nucleotide resolution with a mutant MspA nanopore and phi29 DNA polymerase. Nat. Biotechnol. 30, 349-353 (2012).

20. Peter, C. \& Hummer, G. Ion transport through membrane-spanning nanopores studied by molecular dynamics simulations and continuum electrostatics calculations. Biophys. J. 89, 2222-2234 (2005).

21. Henrickson, S. E., Misakian, M., Robertson, B. \& Kasianowicz, J. J. Driven DNA transport into an asymmetric nanometer-scale pore. Phys. Rev. Lett. 85, 3057-3060 (2000).

22. Meller, A., Nivon, L. \& Branton, D. Voltage-driven DNA translocations through a nanopore. Phys. Rev. Lett. 86, 3435-3438 (2001).

23. Skinner, G. M., van den Hout, M., Broekmans, O., Dekker, C. \& Dekker, N. H. Distinguishing single- and double-stranded nucleic acid molecules using solidstate nanopores. Nano Lett. 9, 2953-2960 (2009).

24. Yeh, I. C. \& Hummer, G. Nucleic acid transport through carbon nanotube membranes. Proc. Natl Acad. Sci. USA 101, 12177-12182 (2004).

25. Lulevich, V., Kim, S., Grigoropoulos, C. P. \& Noy, A. Frictionless sliding of single-stranded DNA in a carbon nanotube pore observed by single molecule force spectroscopy. Nano. Lett. 11, 1171-1176 (2011).

26. Rabin, Y. \& Tanaka, M. DNA in nanopores: Counterion condensation and coion depletion. Phys. Rev. Lett. 94, 148103 (2005).

27. Smeets, R. M. M. et al. Salt dependence of ion transport and DNA translocation through solid-state nanopores. Nano Lett. 6, 89-95 (2006).

28. Booth, M. J. et al. Quantitative sequencing of 5-methylcytosine and 5hydroxymethylcytosine at single-base resolution. Science 336, 934-937 (2012).

29. Song, C.-X. et al. Sensitive and specific single-molecule sequencing of 5hydroxymethylcytosine. Nat. Methods 9, 75-77 (2012).

30. Yu, M. et al. Base-resolution analysis of 5-hydroxymethylcytosine in the mammalian genome. Cell 149, 1368-1380 (2012).

31. Laszlo, A. H. et al. Detection and mapping of 5-methylcytosine and 5-hydroxymethyl-cytosine with nanopore MspA. Proc. Natl Acad. Sci. USA 110, 18904-18909 (2013).

32. Wanunu, M., Morrison, W., Rabin, Y., Grosberg, A. Y. \& Meller, A. Electrostatic focusing of unlabelled DNA into nanoscale pores using a salt gradient. Nat. Nanotech. 5, 160-165 (2010).

33. Wang, Y., Zheng, D. L., Tan, Q. L., Wang, M. X. \& Gu, L. Q. Nanopore-based detection of circulating microRNAs in lung cancer patients. Nat. Nanotech. 6, 668-674 (2011).

34. Wen, S. et al. Highly sensitive and selective DNA-based detection of mercury(II) with $\alpha$-hemolysin nanopore. J. Am. Chem. Soc. 133, 18312-18317 (2011).

35. Mitchell, N. \& Howorka, S. Chemical tags facilitate the sensing of individual DNA strands with nanopores. Angew. Chem. Int. Ed. 47, 5565-5568 (2008).

36. Butler, T. Z., Gundlach, J. H. \& Troll, M. Ionic current blockades from DNA and RNA molecules in the $\alpha$-hemolysin nanopore. Biophys. J. 93, 3229-3240 (2007).

37. Li, W.-W., Gong, L. \& Bayley, H. Single-molecule detection of 5hydroxymethylcytosine in DNA through chemical modification and nanopore analysis. Angew. Chem. Int. Ed. 52, 4350-4355 (2013).

38. Li, X. et al. Selective synthesis combined with chemical separation of singlewalled carbon nanotubes for chirality selection. J. Am. Chem. Soc. 129, 15770-15771 (2007).

39. Tu, X., Manohar, S., Jagota, A. \& Zheng, M. DNA sequence motifs for structure-specific recognition and separation of carbon nanotubes. Nature 460, 250-253 (2009).

40. Holt, J. K. et al. Fast mass transport through sub-2-nanometer carbon nanotubes. Science 312, 1034-1037 (2006).

41. Iijima, S. \& Ichihashi, T. Single-shell carbon nanotubes of 1-nm diameter. Nature 363, 603-605 (1993).

42. Islam, M. F., Milkie, D. E., Torrens, O. N., Yodh, A. G. \& Kikkawa, J. M Magnetic heterogeneity and alignment of single wall carbon nanotubes. Phys. Rev. B 71, 201401 (2005).

43. Liu, J. et al. Fullerene pipes. Science 280, 1253-1256 (1998).

44. Huang, X. Y., McLean, R. S. \& Zheng, M. High-resolution length sorting and purification of DNA-wrapped carbon nanotubes by size-exclusion chromatography. Anal. Chem. 77, 6225-6228 (2005).

45. Holden, M. A. \& Bayley, H. Direct introduction of single protein channels and pores into lipid bilayers. J. Am. Chem. Soc. 127, 6502-6503 (2005).

46. Holden, M. A., Jayasinghe, L., Daltrop, O., Mason, A. \& Bayley, H. Direct transfer of membrane proteins from bacteria to planar bilayers for rapid screening by single-channel recording. Nat. Chem. Biol. 2, 314-318 (2006). 


\section{Acknowledgements}

We thank M. Zheng, M. Chen and T. Zeng for helpful discussions, T.S. Lin, Q. Yuan for assistance with quantitative PCR experiments and T. Li and Y.R. Li for assistance with SWCNT preparation and data analysis. This work was funded by the National Natural Science Foundation of China (numbers 21175135 and 21205119), National Basic Research Program of China (973 programme, numbers 2010СB933600, 2013CB932800 and 2013CB933704) and the '100 Talents' programme of the Chinese Academy of Sciences.

\section{Author contributions}

L.L. and C.Y. performed SWCNT preparation and characterization experiments. C.Y. performed DNA modification experiments. L.L., K.Z. and H.-C.W. performed singlechannel recording experiments. L.L., J.L. and H.-C.W. performed data analysis. L.L. and H.-C.W. conceived the project, designed the experiments and wrote the paper.

\section{Additional information}

Supplementary Information accompanies this paper at http://www.nature.com/ naturecommunications

Competing financial interests: The authors declare no competing financial interests.

Reprints and permission information is available online at http://npg.nature.com/ reprintsandpermissions/

How to cite this article: Liu, L. et al. Ultrashort single-walled carbon nanotubes in a lipid bilayer as a new nanopore sensor. Nat. Commun. 4:2989 doi: 10.1038/ncomms3989 (2013).

(c) (1) (2) This work is licensed under a Creative Commons Attributionc. No NA SA Nommercial-ShareAlike 3.0 Unported License. To view a copy of this license, visit http://creativecommons.org/licenses/by-nc-sa/3.0/ 